\title{
Fault Diagnosis Technology Based on Model Driven
}

\author{
Xie Zhang1, Zufeng Xu1, Jun $\mathrm{Wu}^{2}$ \\ ${ }^{1}$ Research and Development Center, NARI Technology Development Co. Ltd., Nanjing, China \\ ${ }^{2}$ Transmission Maintenance Center, Jiangsu Electric Power Maintenance Branch Company, Nanjing, China \\ Email: zhangxie@sgepri.sgcc.com.cn
}

Received 9 September 2015; accepted 16 October 2015; published 23 October 2015

\begin{abstract}
Fault diagnosis is an important application of the power grids monitoring system. Under the situation of continuous development of smart grid, it brings new challenges to the fault diagnosis technology. A fault diagnosis expert system based on model driven approach is proposed in this paper. And the corresponding fault modeling technology based on Fault Logic Description Language (FLDL) is described step by step. Practices show that this system could meet the requirements of processing fault alarm information rapidly and reliably by operator.
\end{abstract}

\section{Keywords}

Fault Diagnosis, Model Driven, Fault Logic Description Language

\section{Introduction}

Fault diagnosis technology is one of the most important advanced applications in the smart substation [1] [2]. When the fault of power grid happens, it can analyze the related information, find the fault reason and send the fault report to the dispatching center. So it's helpful for the operator to handle the situation. And it is also of great importance for the stable operation of power grid.

So far, various technologies of fault diagnosis have been proposed, such as the method based on the expert system, based on neural network and based on information theory [3]-[7]. The method based on the expert system is the most primary one among them. But it is inflexible to the various fault type which prevents it from being utilized deeply.

In this paper, we studied a new fault diagnosis technology based on model driven. And the corresponding fault modeling technology based on Fault Logic Description Language (FLDL) is also explained step by step. Moreover, an application example in $500 \mathrm{kV}$ Changshu Substation is introduced here.

\section{System Architecture}

The fault diagnosis system based on model driven can be mainly divided into three parts: alarm information process, alarm model resolution and fault reasoning machine. The architecture is shown in Figure 1.

The alarm information process part is the basic service of substation monitoring system. It can receive all the 


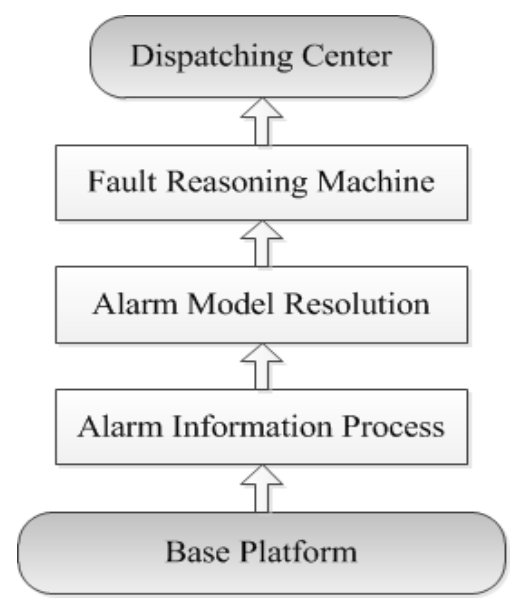

Figure 1. System architecture.

information from the measuring-control and protection devices in real time and divide them into different levels and categories based on rules predefined in the database.

The alarm model resolution part is used to analyze the IEC61850 model information contained in the alarm [8]. After the resolution, the related information about the secondary device and the corresponding primary device can be obtained. Also the matched fault model can be prepared for the further reasoning process.

The fault reasoning machine is the key part of fault diagnosis. It receives the alarm information from the alarm resolution part and compares it with all the constraint conditions stated in the fault model. When all the fault characteristics and the constraint conditions are satisfied, the corresponding result will be concluded and the reasoning report will be sent to the dispatching center.

\section{Substation Fault Modeling Technology Based on FLDL}

The fault model is the basis of substation fault diagnosis expert system. Based on the unified device model, the fault logic model can be abstracted from various fault logic. And Fault Logic Description Language is adopted to describe the model.

\subsection{Substation Fault Logic Model}

The substation fault is basically divided into two categories: secondary equipment fault and power grid fault. The fault logic of secondary equipment is mainly based on the alarm information and the measurement information, which represents a type of fault individually. The power grid fault relates to the voltage level, connection mode and the fault scale. According to the summarization of typical fault characteristics, a group of production rules can be concluded. Take a certain $220 \mathrm{kV}$ bus fault as an example, the fault logic is shown in Figure 2. In this figure, the breakers of chunshen I, chunshen II, weitang I, weitang II and the bus tie breaker are all the related breakers of II bus. Based on its typical characteristics, we can obtain the general fault logic of 220kV bus fault as shown in Figure 3.

After the fault logic model has been concluded, a standardized format should be built to meet the requirements of reading and maintenance. The former expert system is limited to use because of its fixed structure. In this paper, Fault Logic Description Language (FLDL) which can be recognized by the computer is proposed to describe the typical fault logic model.

\subsection{Fault Logic Description Language}

Fault Logic Description Language (FLDL) complies with Extensible Markup Language (XML) specification. It takes advantage of XML specification which is universal, expandable and readable. FLDL can completely describe the substation fault characteristics including the constraint conditions such as the voltage level, phase and so on, which brings benefits to the fault reasoning. The fault model structure using FLDL is shown in Figure 4. The basic structure of FLDL is the element, which has different attributes. 


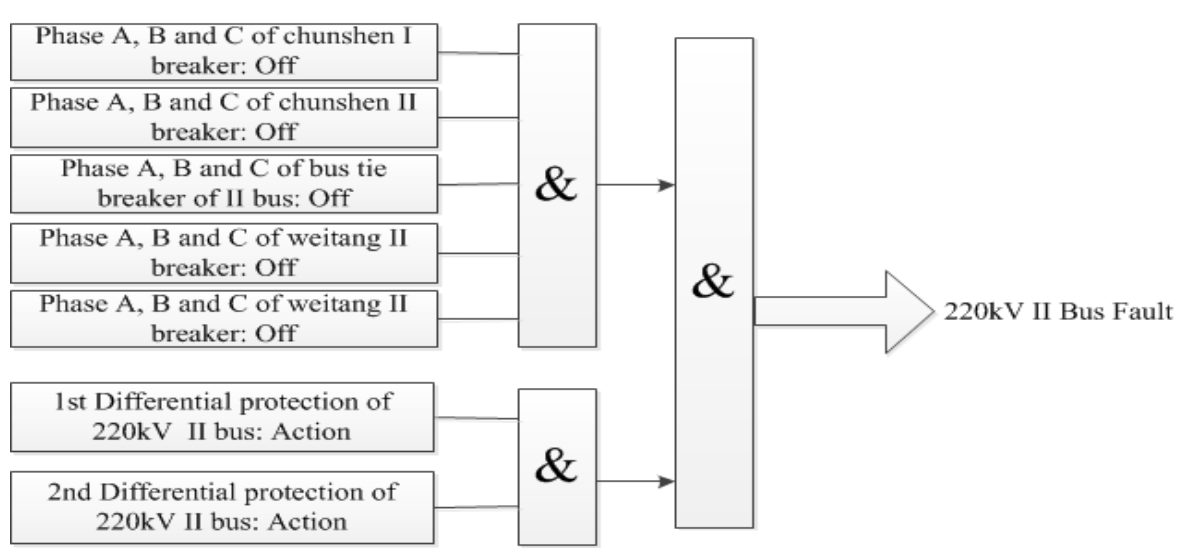

Figure 2. Example of a certain $220 \mathrm{kV}$ bus fault.

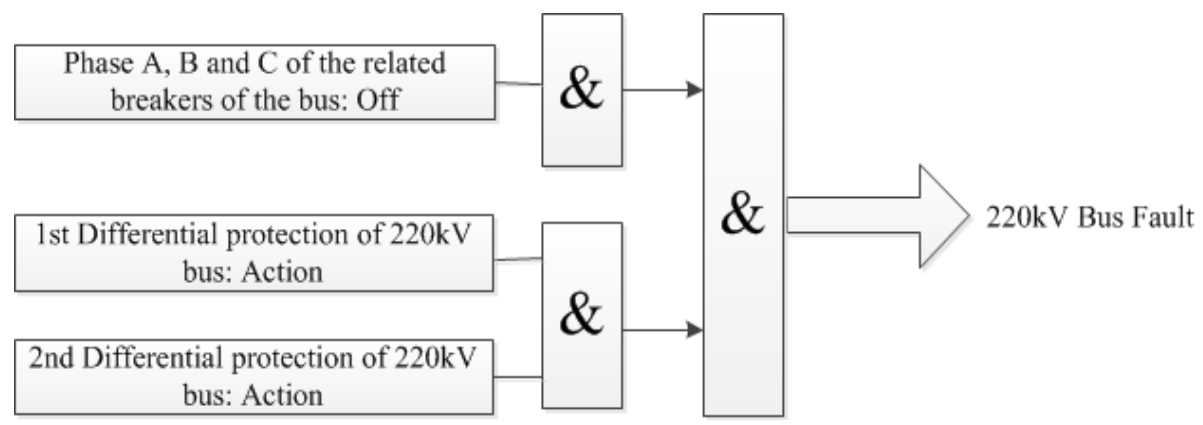

Figure 3. General fault logic of $220 \mathrm{kV}$ bus fault.

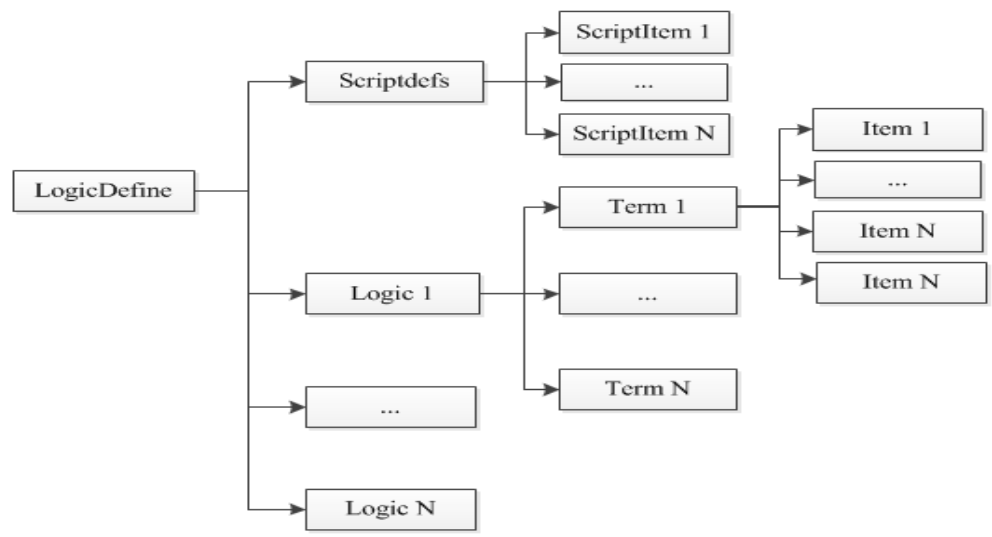

Figure 4. Fault model structure using FLDL.

The root element LogicDefine is the parent object of the other elements.

Taking into account of the arithmetic logical operation involved in the fault reasoning, the method of script calculate is adopted. The script formula is stored in the script file in advance. Scriptdefs element is the sub-element of LogicDefine. It defines the script files which will be quoted in FLDL. And ScriptItem is the sub-element of Scriptdefs which describes the path of the quoted script files.

Logic is also the sub-element of LogicDefine which defines a specific fault model as shown in Figure 3. Logic element describes the fault attributes such as voltage level, phase, scale and timeout.

Term is the sub-element of Logic, which is also the basic unit of fault model. It contains the attributes of launch flag, logic sequence, quoted script and so on. Term consists of a group of Items, which is the smallest unit of fault model. Moreover, Item is divided into LogicItem and CalcItem based on the functional requirements. LogicItem has the attributes of data object, data source and data type, while CalcItem marks the attributes 
of quoted script and so on. In addition, as the sub-element of CalcItem, DataItem also contains the attributes of data object and data source which is used to define the data needed by CalcItem.

FLDL introduced above adopts the multi-level keyword structure to describe the involved device object and data object in the model as shown in Figure 4. It can not only meet the functional requirements of fault logic, but also can be understood easily by the computer and people.

\section{Fault Diagnosis Technology by Model Driven}

The former fault diagnosis expert system usually adopts the data driven method which binds the code directly with the data [3]. This method can help to process the fault alarm information rapidly. But its inflexible to the variable demand of fault logic holds back the progress of fault diagnosis technology. However, the fault diagnosis by model driven can solve the problem effectively.

The key of model driven method is to load the standard model dynamically instead of binding it to the code.

As we can see from Figure 5, the reasoning machine receives the alarm information from the resolution module and compares it with the launch condition of fault reasoning instance. When they match, the following reasoning step will be carried out. If all the fault characteristics and the constraint conditions are satisfied, the fault reasoning completes and the corresponding result will be concluded. Finally, the reasoning result will be sent to the dispatching center.

The model driven method separates the fault logic and the program, which can simplify the process of reasoning machine and improve the flexibility in application. Meanwhile, the whole process of reasoning machine is divided into three parts: the main flow of reasoning machine, the launch flow of logic instance and the process flow of logic instance. This method simplifies the complicated fault diagnosis process and also improves the efficiency of reasoning machine.

\section{Application}

The fault diagnosis system based on model driven is already in use in 500kV Changshu Substation in Suzhou.

Take the transient fault of transmission line as an example. The Sequence of Event (SOE) alarm information received by the alarm window is shown in Table 1. And the reasoning result is also shown in the alarm window as illustrated in Figure 6. Figure 7 shows the corresponding fault report window. Meanwhile, the reasoning result and the fault report will be sent to the dispatching center via service bus for further analysis.

From the practical operation, the fault reasoning process can be completed in the fault period (time from the beginning to the end of the fault, usually 7 - 8 s) and send the fault report to the dispatching center. Compared with the traditional analysis method, the fault diagnosis by model driven is quicker, more accurate and more reliable. It can not only deal with the typical fault, but also meet the requirements of complicated fault type. Moreover, the fault time, fault device, fault phase and the corresponding bay contained in the report is helpful for the operator to judge quickly and avoid the continuous expansion of influence.

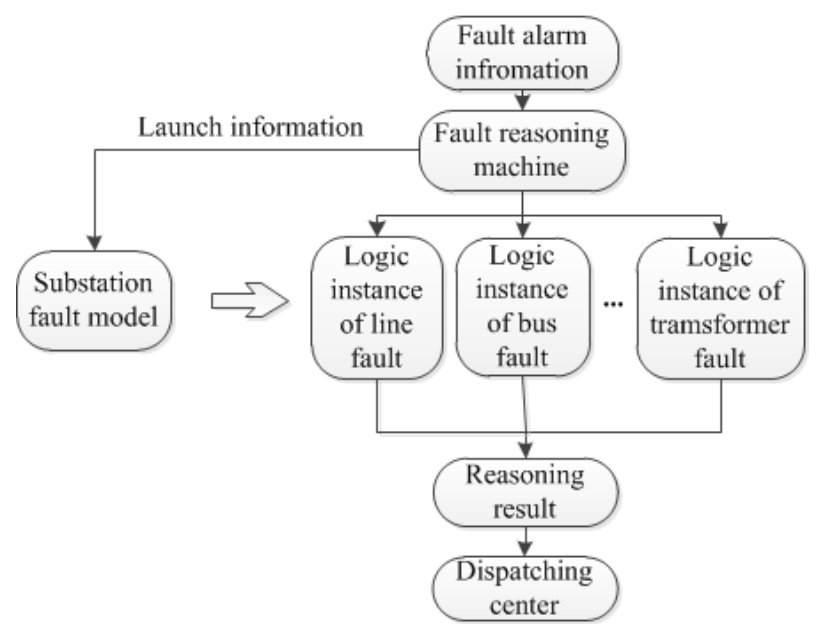

Figure 5. Process flow of fault diagnosis technology by model driven. 


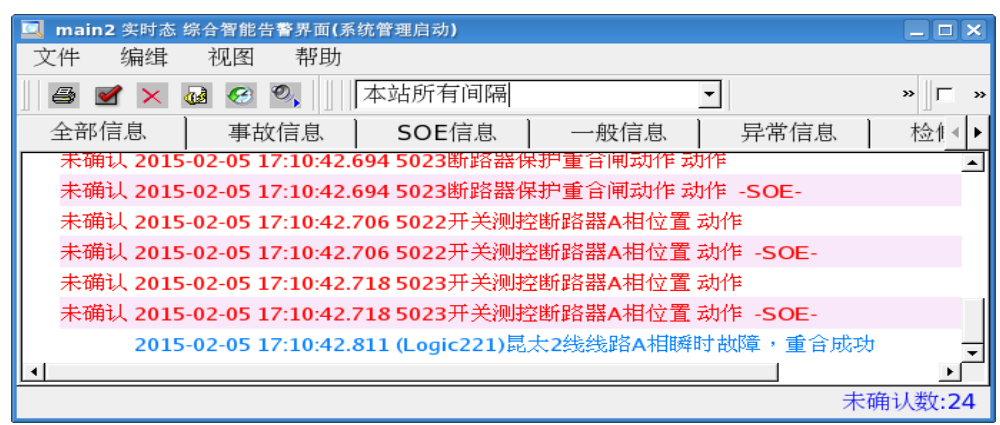

Figure 6. Reasoning result in the alarm window.

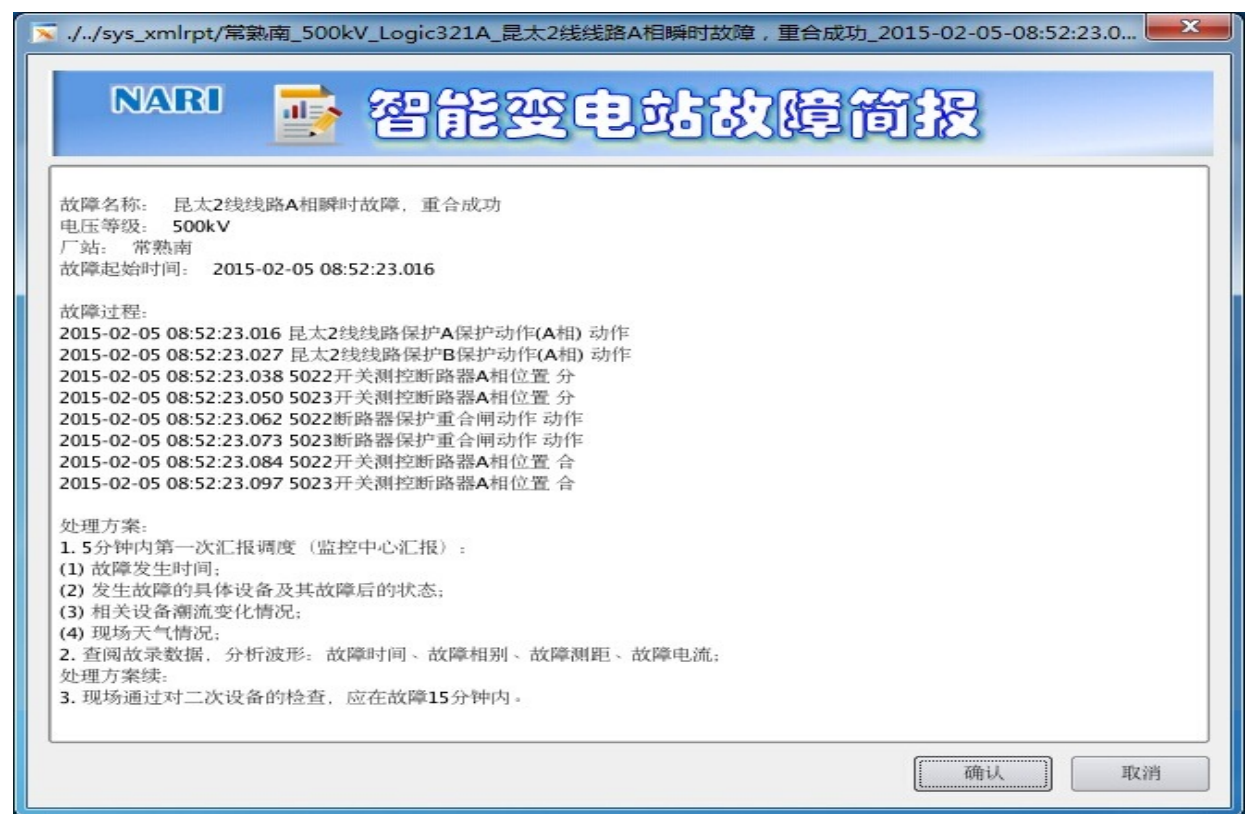

Figure 7. Fault report window.

Table 1. SOE alarm information received by alarm window.

\begin{tabular}{cc}
\hline Time $(\mathrm{ms})$ & Alarm Information \\
\hline 0 & Line protection of kuntai 2 (Phase A): Action \\
11 & Line protection of kuntai 2 (Phase B): Action \\
22 & Breaker of 5022 measuring-control device (Phase A): Off \\
34 & Breaker of 5023 measuring-control device (Phase A): Off \\
46 & Reclose of 5022 protection device: Action \\
57 & Reclose of 5023 protection device: Action \\
68 & Breaker of 5022 measuring-control device (Phase A): On \\
81 & Breaker of 5023 measuring-control device (Phase A): On \\
\hline
\end{tabular}

\section{Conclusion}

In this paper, we proposed the fault diagnosis technology by model driven and the corresponding fault modeling technology based on Fault Logic Description Language (FLDL) step by step. It is practically adopted in $500 \mathrm{kV}$ Changshu Substation in Suzhou. The result shows that it can help to analyze the fault reason rapidly and reliably so that the operator can deal with it in time. And it also makes the fault diagnosis application flexible, applicable and accurate for higher practicability and standardization. This method is of great importance for the further study. 


\section{References}

[1] Wei, L.H., Guo, W.X. and Wen, F.S. (2011) An Online Intelligent Alarm-Processing System for Digital Substations. IEEE Transactions on Power Delivery, 26, 1615-1624. http://dx.doi.org/10.1109/TPWRD.2011.2117445

[2] Kezunovic, M., Guan, Y.F. and Guo, C.Y. (2010) The 21st Century Substation Design: Vision of the Future. 2010 IREP Symposium-Bulk Power System Dynamics and Control VIII (IREP), Buzios, 1-6 August 2010. http://dx.doi.org/10.1109/irep.2010.5563267

[3] Lee, H.J., Ahn, B.S. and Park, Y.M. (2000) A Fault Diagnosis Expert System for Distribution Substations. IEEE Trans on Power Delivery, 15, 92-97. http://dx.doi.org/10.1109/61.847234

[4] Cardoso, G., Rolim, J.G. and Zurn, H.H. (2004) Application of Neural-Network Modules to Electric Power System Fault Section Estimation. IEEE Trans on Power Delivery, 19, 1034-1041. http://dx.doi.org/10.1109/TPWRD.2004.829911

[5] Mahanty, R.N. and Gupta, P.B.D. (2004) Application of RBF Neural Network to Fault Classification and Location in Transmission Lines. IET Proceedings of Generation, Transmission \& Distribution, 151, 201-212. http://dx.doi.org/10.1049/ip-gtd:20040098

[6] Srinivasan, D., Cheub, R.L., Poh, Y.P. and Ng, A.K.C. (2000) Automated Fault Detection in Power Distribution Networks Using a Hybrid Fuzzy-Genetic Algorithm Approach. Engineering Applications of Articial Intelligence, 13, 407418. http://www.sciencedirect.com/science/article/pii/S0952197600000129

[7] Bedekar, P.P., Bhide, S.R. and Kale, V.S. (2011) Fault Section Estimation in Power System Using Hebb’s Rule and Continuous Genetic Algorithm. International Journal of Electrical Power and Energy System, 33, 457-465. http://dx.doi.org/10.1016/j.ijepes.2010.10.008

[8] Schwarz, K. (2002) Standard IEC61850 for Substation Automation and Other Power System Applications. Power Systems and Communications Infrastructures for the Future, Beijing, September 2002. 